\title{
Comment on "A New Look at Some Old Data: The Beveridge Wheat Price Series" by Granger and Hughes
}

\author{
By Ronald LeE
}

\section{University of Michigan}

THERE is a small but potentially important error in this useful and interesting paper by Granger and Hughes (1971). The paper analyses the effects on the estimated spectrum of dividing each original observation by a centred moving average of length $2 m+1$ terms. While the mathematical and numerical results are correct, the discussion of the effects of the transformation is seriously misleading.

An appendix to the paper establishes that the effect of the transform is approximately described by the squared gain function:

$$
A(w)=\left\{1-\sin \left(m+\frac{1}{2}\right) w /(2 m+1) \sin (w / 2)\right\}^{2} .
$$

The discussion on p. 418 incorrectly asserts that $A(w)$ will reach peak values of unity for $w$ corresponding to periods of $2 m+1,(2 m+1) / 2,(2 m+1) / 3$, etc., years. Since Beveridge used a 31 -year moving average and found a 15.5 -year cycle, the suspicion that the transformation created the cycle arises. However, a simple inspection of (1.1) shows it will attain values greater than unity when the ratio of sines is negative. After calculating $d A(w) / d w$ and using the approximation $\tan (x) \doteq x$ for small $x$ measured in radians, it can be shown that $A(w)$ actually peaks at periods slightly longer than $2 m+1$ times factors of $2 / 3,2 / 7,2 / 11$, etc., rather than factors of $1,1 / 2,1 / 3$ as claimed by the authors. For Beveridge's 31-year moving average, the peaks are at $21 \cdot 8,8 \cdot 95,5 \cdot 65,4 \cdot 14$, etc., years, and there is no tendency to amplify a $15 \cdot 5$-year cycle.

Having pointed this out, I hasten to add that the authors' numerical results are not affected; apparently their calculated $A(w)$ was correct. The whole point would not be worth mentioning except that others, like myself, may rely on the described effect of the filter. After using a 61-year moving average to detrend a series of plague outbreaks, I found a strong 45-year spectral peak. The Granger and Hughes article convinced me it was real, since spurious peaks might be expected at periods of 61 or 30.5 years, but not 45 years; it was only by chance that I tried "recolouring" and found that it then disappeared. The true peak amplification was at a period of about 42 years, or slightly more than $(2 / 3)(61)=40 \cdot 7$.

\section{REFERENCE}

Granger, C, W. J. and Hughes, A. O. (1971). A new look at some old data: the Beveridge wheat price series. J. R. Statist. Soc. A, 134, 413-428.

\section{Correction}

IN the Discussion on the Report of the Joint Committee on Teaching Statistics which was published in this Journal, Vol. 137 (1974), on p. 422 the contribution by Mr P. C. WeBs (Department of Education and Science) was incorrectly attributed to the name Wells. We apologize for this oversight. 